\title{
Comparison of three-dimensional quantitative coronary angiography and intravascular ultrasound for detecting functionally significant coronary lesions
}

\author{
Jooho Lee ${ }^{1}$, Kyoung-Woo Seo ${ }^{2}$, Hyoung-Mo Yang ${ }^{2}$, Hong-Seok Lim ${ }^{2}$, Byoung-Joo Choi ${ }^{2}$, So-Yeon Choi ${ }^{2}$, \\ Seung-Jae Tahk ${ }^{2}$, Myeong-Ho Yoon ${ }^{2}$ \\ ${ }^{1}$ Division of Cardiology, Department of Internal Medicine, Seoul Medical Center, Seoul, Korea; ${ }^{2}$ Department of Cardiology, Ajou University School \\ of Medicine, Suwon, Korea \\ Contributions: (I) Conception and design: HM Yang, J Lee; (II) Administrative support: HM Yang; (III) Provision of study materials or patients: KW \\ Seo, HM Yang, HS Lim, BJ Choi, SY Cho, SJ Tahk; (IV) Collection and assembly of data: KW Seo, HM Yang, HS Lim, BJ Choi, SY Cho, SJ Tahk; (V) \\ Data analysis and interpretation: HM Yang, J Lee; (VI) Manuscript writing: All authors; (VII) Final approval of manuscript: All authors. \\ Correspondence to: Myeong-Ho Yoon, MD, PhD. Department of Cardiology, Ajou University School of Medicine, 164, Worldcup-Ro, Yeongtong-gu, \\ Suwon, 16499, Korea. Email: yoonmh65@hanmail.net.
}

\begin{abstract}
Background: Three-dimensional quantitative coronary angiography (3D-QCA) can provide more accurate measurement of true vessel size and may be comparable to intravascular ultrasound (IVUS) in identifying functionally significant coronary stenosis, as determined by fractional flow reserve (FFR). This study aimed to evaluate the diagnostic accuracy of 3D-QCA for predicting FFR $<0.8$.

Methods: We assessed 175 lesions in 175 patients by FFR, IVUS, and 3D-QCA. Correlations between 3D-QCA values, IVUS values, and FFR values were analyzed. Receiver operating characteristic (ROC) curves were used to evaluate diagnostic accuracy of 3D-QCA for predicting FFR $<0.8$ and to determine the appropriate cut-off value.
\end{abstract}

Results: Upon evaluating 3D-QCA values, minimum lumen area (MLA) correlated with FFR value ( $r=0.48$, $\mathrm{P}<0.001)$. Considering IVUS values, MLA correlated with FFR value $(\mathrm{r}=0.43, \mathrm{P}<0.001)$. Also, 3D-QCA MLA was well correlated with IVUS MLA ( $r=0.61, \mathrm{P}<0.001)$. The area under the ROC curve (AUC) for 3D-QCA MLA was 0.77, and the best cut-off value was 2.37 (sensitivity: 73\%, specificity: 71\%). The AUC for IVUS MLA was 0.73 , and the best cut-off value was 3.01 (sensitivity: $71 \%$, specificity: $65 \%$ ). There was no significant difference in AUC for 3D-MLA and IVUS-MLA $(\mathrm{P}=0.27)$.

Conclusions: 3D-QCA is not inferior to IVUS for functional assessment of intermediate coronary lesions. We can consider 3D-QCA as a suitable substitute for IVUS or FFR in determining coronary intervention.

Keywords: Coronary artery disease; fractional flow reserve (FFR); quantitative coronary angiography; intravascular ultrasound (IVUS)

Submitted Jun 09, 2020. Accepted for publication Aug 30, 2020.

doi: $10.21037 / \mathrm{cdt}-20-560$

View this article at: http://dx.doi.org/10.21037/cdt-20-560

\section{Introduction}

Fractional flow reserve (FFR) can identify ischemiaproducing lesions with a cut-off value of FFR $<0.80$ and is regarded as the standard of reference for evaluation of functionally significant coronary lesions $(1,2)$. Intravascular ultrasound (IVUS) can provide more detailed assessment of lumen morphology and lesion severity than visual estimation on two-dimensional quantitative coronary angiography (2D-QCA). Recent studies have suggested the optimal IVUS criteria for predicting ischemic FFR value $<0.80$. FFR and IVUS provide additional information for physiological and anatomical lesion significance but require 

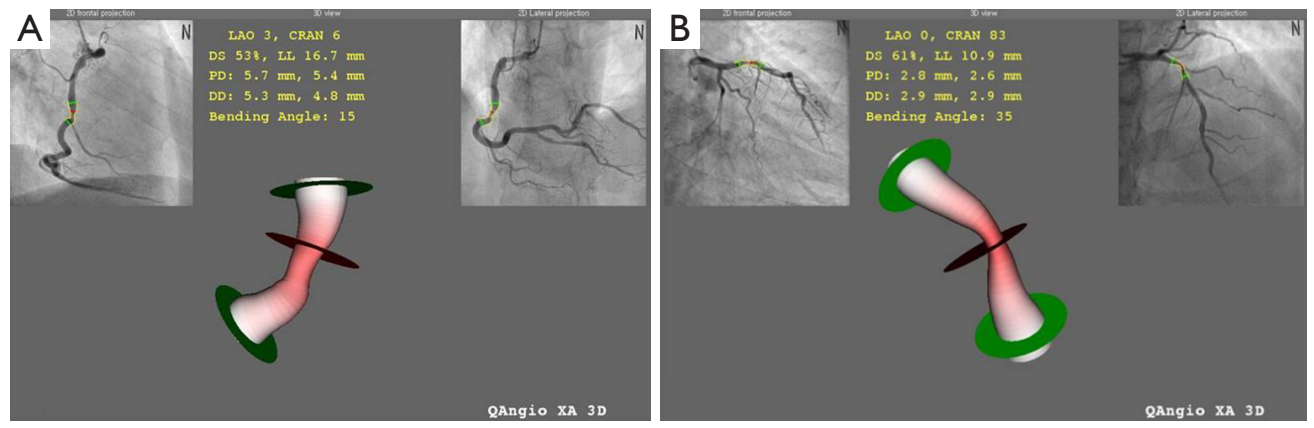

Figure 1 Examples of 3D-QCA of right coronary artery (A) and left anterior descending artery (B) using two projections. QCA, quantitative coronary angiography.

a wiring procedure and time (3-7). Three-dimensional quantitative coronary angiography (3D-QCA) derived from routine $2 \mathrm{D}$ angiography has been developed and offers a reasonable alternative to FFR or IVUS (8-13). The purpose of this study was to compare the diagnostic efficacy of 3D-QCA, and IVUS for detecting functionally significant coronary lesions determined by FFR $<0.80$. We present the following article in accordance with the STARD reporting checklist (available at http://dx.doi.org/10.21037/cdt-20560).

\section{Methods}

\section{Study population}

Between April 2011 and August 2014, we included 175 patients with stable ischemic heart disease who underwent coronary angiography and revealed intermediate severity (50-70\% on visual estimation) at Ajou university hospital. All patients had both FFR and IVUS examination. 175 lesions in 175 patients were assessed retrospectively by FFR, IVUS, 2D-, and 3D-QCA. Exclusion criteria were tandem lesion in the target vessel (stenosis $>50 \%$ of diameter on visual estimation), prior myocardial infarction, prior coronary artery bypass surgery, prior implanted stent in the target vessel, and acute coronary syndrome. We also excluded myocardial bridge defined by difference of diameter stenosis (DS)\% higher than 20\% between cardiac cycles by visual estimation. The study was conducted in accordance with the Declaration of Helsinki (as was revised in 2013) and the Harmonized Tripartite Guideline for Good Clinical Practice from the International Conference on Harmonization. This study was reviewed and approved by Ajou University Hospital Institutional Review Board (AJIRB-MED-MDB-20). Because of the retrospective nature of the research, the requirement for informed consent was waived.

\section{FFR measurement}

The 0.014-inch pressure guide wire (Radi, St Jude Medical, Uppsala, Sweden) was externally calibrated, and "equalizing" was performed at the guiding catheter tip. The pressure guide wire was positioned distal to the lesion. FFR was measured at maximal hyperemia induced by intravenous adenosine infusion $(140 \mu \mathrm{g} / \mathrm{kg} / \mathrm{min})$ or intracoronary adenosine bolus infusion $(60-80 \mu \mathrm{g})$. An FFR value $<0.80$ was considered functionally significant. On operator discretion, the lesion was treated by percutaneous coronary intervention.

\section{Quantitative coronary angiography}

2D-QCA analysis was performed with standard automated edge-detection function (CASS-5, Pie Medical, Masstricht, Netherlands). 3D-QCA analysis was performed blinded to the FFR and IVUS data using QAngio XA 3D (Medis Medical Imaging Systems, Leiden, Netherlands). For $3 \mathrm{D}-\mathrm{QCA}$ analysis, two angiographic images at least $25^{\circ}$ apart were selected. Lumen contours were delineated for each projection using the automated edge-detection function as used in 2D-QCA. After processing lumen contours, coronary lumen and reference vessel were reconstructed as shown in Figure 1.

\section{IVUS imaging and analysis}

IVUS studies was performed after administration of $0.2 \mathrm{mg}$ intracoronary nitroglycerin using motorized 
Table 1 Baseline clinical characteristics of 175 patients

\begin{tabular}{lc}
\hline Characteristics & Data \\
\hline Age (years) & $66 \pm 15$ \\
Male & $110(63 \%)$ \\
Cardiac risk factors & \\
Hypertension & $98(56 \%)$ \\
Dyslipidemia & $31(18 \%)$ \\
Diabetes mellitus & $45(26 \%)$ \\
Current smoker & $40(23 \%)$ \\
Family history & $13(7 \%)$ \\
\hline
\end{tabular}

transducer pullback $(0.5 \mathrm{~mm} / \mathrm{s})$ and a commercial scanner (Boston Scientific/SCIMED, Minneapolis, MN) consisting of a rotating $40-\mathrm{MHz}$ transducer within a $3.2 \mathrm{~F}$ imaging sheath. IVUS images were analyzed by 2 trained cardiologists in our center blinded to the FFR results using computerized plannimetry (EchoPlaque 3.0, Indec Systems, Mountain View, CA). The proximal and distal reference segments were selected within $10 \mathrm{~mm}$ proximal and distal to the lesion without any side branches. Minimum lumen area (MLA) was measured by tracing the border of echo-dense plaque at the site of the smallest lumen, and plaque burden $(\mathrm{PB})$ at the MLA site was calculated as [external elastic membrane $($ EEM) area - lumen area)/EEM area $\times 100(\%)]$. Lesion length was measured from the most proximal to the most distal site of stenosis.

\section{Statistical analysis}

All statistical analyses were performed using SPSS version 20.0 (SPSS Inc, Chicago, IL) and MedCalc version 14.8 (MedCalc Software, Ostend, Belgium). All values were expressed as mean \pm standard deviation (continuous variables) or as number and percentage (categorical variables). Receiver operating characteristic (ROC) curve analysis was used to establish the cut-off values of IVUS and QCA parameters for predicting FFR $<0.8$. The best cutoff values were calculated using the Youden index. The area under the ROC curve (AUC) was compared by using the DeLong test.

\section{Results}

The baseline clinical characteristics and the angiographic and IVUS measurements in 175 patients with 175 lesions are summarized in Tables 1,2. The mean FFR value at hyperemia was 0.81 , and 70 vessels $(40 \%)$ showed FFR $<0.8$. The mean difference of 3D-QCA MLA and IVUS MLA was 0.66 [95\% confidence interval (CI), 0.47 to 0.86] (Figure 2). The MLA of 3D-QCA correlated with FFR value $(\mathrm{r}=0.48, \mathrm{P}<0.001)$ (Figure 3). Of IVUS values, MLA correlated with FFR value $(\mathrm{r}=0.43, \mathrm{P}<0.001)$ (Figure 4). Also, 3D-QCA MLA was well correlated with IVUS MLA $(\mathrm{r}=0.61, \mathrm{P}<0.001)$ (Figure 5). The ROCs analysis for predicting FFR $<0.8$ is shown in Table 3. The area under the ROC curve (AUC) for 3D-QCA DS\% was 0.72 , and the best cut-off value was 51.3 (sensitivity: 67\%, specificity: $65 \%$, accuracy: $66 \%$ ). The AUC for 3D-QCA MLA was 0.77 , and the best cut-off value was 2.37 (sensitivity: $73 \%$, specificity: $71 \%$, accuracy: $73 \%$ ). The AUC for IVUS MLA was 0.73 , and the best cut-off value was 3.01 (sensitivity: $71 \%$, specificity: $65 \%$, accuracy: $68 \%$ ) (Figure 6). There was no significant difference in AUC for 3D-QCA DS\%, and 3D-QCA MLA with IVUS-MLA $(\mathrm{P}=0.89$ and $\mathrm{P}=0.27$, respectively).

\section{Discussion}

This study aimed to directly compare the 3D-QCA and IVUS for predicting functionally significant coronary lesions determined by FFR $<0.80$. We demonstrate that 3D-QCA offers comparable diagnostic efficacy to IVUS for detecting FFR $<0.80$.

\section{IVUS and FFR in assessment of coronary stenosis}

Visual estimation and conventional 2D-QCA have been routinely used for assessing coronary disease for a long time (8). However, it is difficult to depict true vascular structures of coronary arteries due to limited spatial and temporal resolutions, inability to reflect extraluminal irregularities, lesion asymmetry, and tortuosity. Intracoronary imaging modality like IVUS has been widely used to overcome anatomical pitfalls of visual estimation and 2D-QCA $(9,11,12,14)$. In addition to the anatomical significance obtained by IVUS, FFR may confer additional information of the physiological significance of coronary stenosis. Although it is not possible to determine the exact ischemic potential of epicardial stenosis only based upon vessel geometry, some correlation between FFR and stenosis geometry exists $(4,5,7,10,15-18)$. Several studies identified IVUS cut-off values of 4 and $6 \mathrm{~mm}^{2}$ for a MLA when 
Table 2 QCA, IVUS, and FFR measurements in 175 lesions

\begin{tabular}{|c|c|}
\hline Measurements & Data \\
\hline \multicolumn{2}{|l|}{ Vessel } \\
\hline Left anterior descending artery & $150(86 \%)$ \\
\hline Left circumflex artery & $7(4 \%)$ \\
\hline Right coronary artery & $18(10 \%)$ \\
\hline \multicolumn{2}{|l|}{ 2D-QCA } \\
\hline Diameter stenosis, \% & $53.5 \pm 13.30$ \\
\hline Minimum lumen diameter, mm & $1.5 \pm 0.5$ \\
\hline Reference vessel diameter, $\mathrm{mm}$ & $3.2 \pm 0.6$ \\
\hline Lesion length, mm & $21.9 \pm 11.1$ \\
\hline \multicolumn{2}{|l|}{ 3D-QCA } \\
\hline Minimum lumen area, $\mathrm{mm}^{2}$ & $2.78 \pm 1.23$ \\
\hline Reference vessel area, $\mathrm{mm}^{2}$ & $8.04 \pm 3.72$ \\
\hline Lesion length, mm & $18.08 \pm 8.13$ \\
\hline Diameter stenosis, \% & $47.6 \pm 12.50$ \\
\hline Percent area stenosis, \% & $55.56 \pm 14$ \\
\hline \multicolumn{2}{|l|}{ IVUS } \\
\hline Minimum lumen area, $\mathrm{mm}^{2}$ & $3.44 \pm 1.4$ \\
\hline EEM area at MLA site, $\mathrm{mm}^{2}$ & $11.81 \pm 3.77$ \\
\hline Plaque burden, \% & $69 \pm 11$ \\
\hline Proximal reference lumen area, $\mathrm{mm}^{2}$ & $12.19 \pm 11.12$ \\
\hline Proximal reference EEM area, $\mathrm{mm}^{2}$ & $16.21 \pm 3.67$ \\
\hline Distal reference lumen area, $\mathrm{mm}^{2}$ & $8.49 \pm 3.41$ \\
\hline Distal reference EEM area, $\mathrm{mm}^{2}$ & $11.83 \pm 4.25$ \\
\hline Lesion length, mm & $23.42 \pm 11.23$ \\
\hline \multicolumn{2}{|l|}{ FFR } \\
\hline Pre-adenosine & $0.92 \pm 0.05$ \\
\hline Post-adenosine & $0.81 \pm 0.07$ \\
\hline
\end{tabular}

QCA, quantitative coronary angiography; MLA, minimum lumen area; IVUS, intravascular ultrasound; FFR, fractional flow reserve.

differentiating functionally significant coronary lesions in non-left main coronary artery and left main coronary artery, respectively (4-6,18-20). In a study by Kang et al., the best cut-off value of the MLA to predict FFR $<0.80$ was $2.4 \mathrm{~mm}^{2}$ with a diagnostic accuracy of $68 \%$ (3). Koo et al. raised the importance of reference vessel size by reporting the segment-specific cut-off value of $3.0 \mathrm{~mm}^{2}$ for proximal

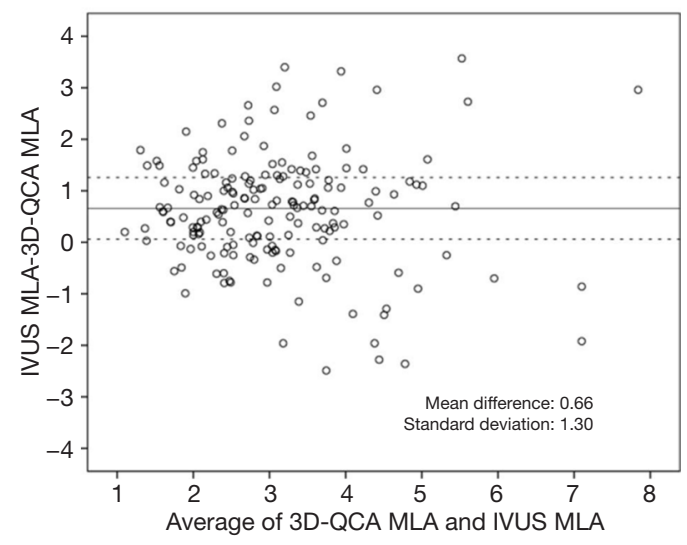

Figure 2 Difference between 3D-QCA MLA and IVUS MLA. QCA, quantitative coronary angiography; MLA, minimum lumen area; IVUS, intravascular ultrasound.

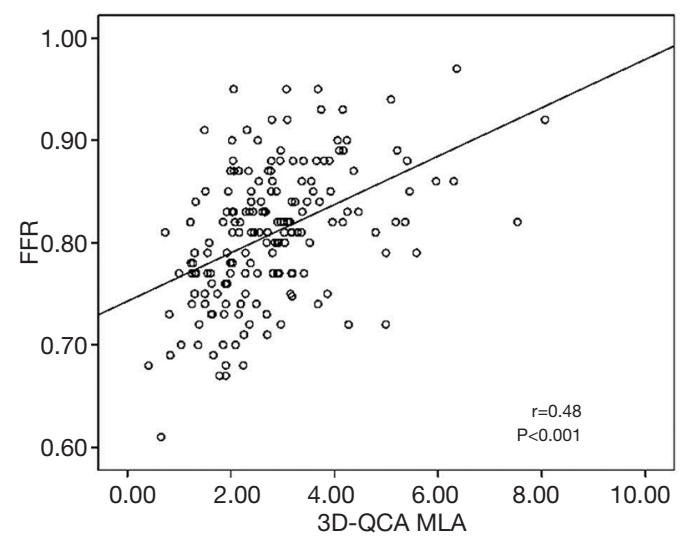

Figure 3 Linear correlation analysis of MLA by 3D-QCA and FFR. QCA, quantitative coronary angiography; MLA, minimum lumen area; FFR, fractional flow reserve.

LAD lesions and $2.75 \mathrm{~mm}^{2}$ for mid LAD lesions (21).

\section{Utility of $3 D-Q C A$}

3D-QCA has been recently developed to compensate for the limitations of conventional 2D-QCA and allows accurate delineation of true vessel structure by fusion of two angiographic views $(8,12-14,22)$. Even though 3D-QCA is derived from incomplete $2 \mathrm{D}$ luminography and cannot fully overcome the limitations of 2D-QCA, it may be able to better reflect vessel tortuosity and lesion eccentricity in the assessment of coronary lesions. 3D-QCA can be more easily obtained from preexisting orthogonal 2D images than 
can FFR or IVUS. Nishi et al. have reported that 3D-QCA parameters have better predictive value for reduced FFR compared with 2D-QCA. In that study, 3D-QCA MLA and MLD utilized for reduced FFR were comparable to IVUS MLA of 42 lesions (11).

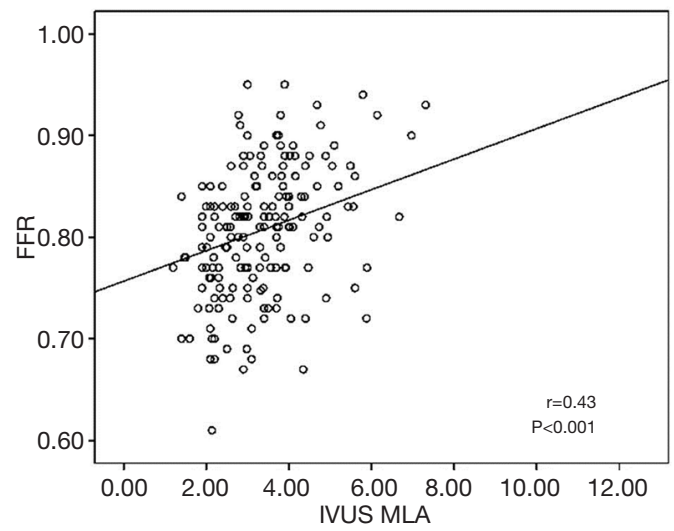

Figure 4 Linear correlation analysis of MLA by IVUS and FFR. MLA, minimum lumen area; IVUS, intravascular ultrasound; FFR, fractional flow reserve.

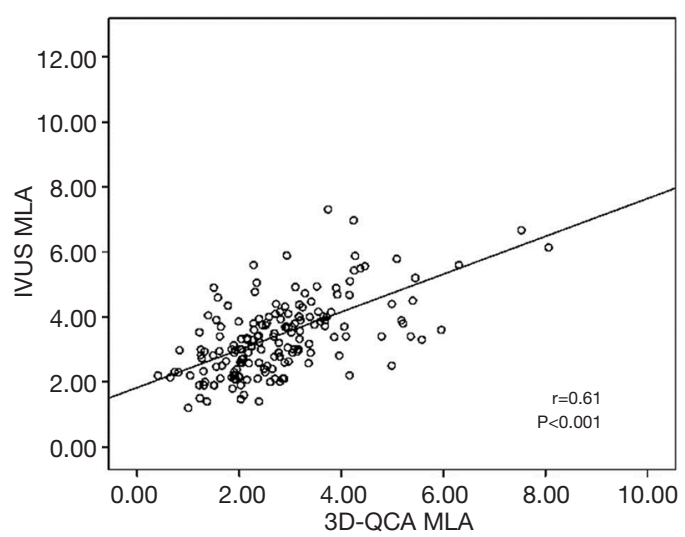

Figure 5 Linear correlation analysis of MLA by 3D-QCA and MLA by IVUS. QCA, quantitative coronary angiography; MLA, minimum lumen area; IVUS, intravascular ultrasound.

\section{Comparison of $3 D-Q C A$ and IVUS for reduced FFR}

The present study aimed to reinforce T. Nishi's results with enrollment of many more coronary lesions. Anatomical value of 3D-QCA MLA, and IVUS MLA was correlated with physiologic value of FFR. 3D-QCA MLA and IVUS MLA showed linear correlation with each other. Nishi et al. suggested that the diagnostic accuracy of 3D-QCA MLA for reduced FFR using ROC analysis was comparable to that of IVUS MLA (11). Huang et al. reported that diagnostic accuracy of 3D-QCA derived DS\% for reduced FFR was $75 \%$ (23). Similarly, in our study, the diagnostic accuracy of 3D-QCA DS\%, 3D-QCA MLA, and IVUS MLA for ischemic FFR was $66 \%, 73 \%$, and $68 \%$. Diagnostic accuracy of 3D-QCA DS\% is slightly lower than that of 3D-QCA MLA and IVUS MLA. The area parameter may reflect much truer vessel geometry than diameter parameter. The best cut-off value of 3D-QCA DS\%, 3D-QCA MLA, and IVUS MLA for ischemic FFR was $51.3 \%, 2.37 \mathrm{~mm}^{2}$ and $3.01 \mathrm{~mm}^{2}$, respectively. In other IVUS studies, 2D-QCA derived MLA that was calculated from MLD showed a mean difference of $1-1.5 \mathrm{~mm}^{2}$ from IVUS MLA $(4,9,19,21)$. The mean difference of 3D-QCA MLA and IVUS MLA in our study was $0.66 \mathrm{~mm}^{2}$, and this smaller difference may be due to correction effect by 3D-QCA. AUC of 3D-QCA MLA and IVUS MLA for predicting FFR $<0.80$ showed no statistically significant difference, implicating similar diagnostic efficacy. In the study of Huang et al., optical coherence tomography (OCT) based FFR showed superiority in predicting ischemic FFR than 3D-QCA derived parameters, integrating both anatomical and physiologic significance. We also integrated both anatomical and physiologic parameter by validating the optimal cut off value (23). In real world practice, FFR and IVUS are relatively expensive tools and require additional procedure and time, though they have many advantages. If FFR or IVUS are not available, 3D-QCA may be a good substitute in assessment of intermediate coronary stenosis without extra procedures (8).

Table 3 Receiver operating characteristics curve analysis for predicting FFR $<0.8$

\begin{tabular}{lccccc} 
& Best cut-off & Sensitivity & Specificity & AUC & Accuracy \\
\hline 3D-QCA DS\% & $51.3 \%$ & $67 \%$ & $65 \%$ & 0.72 & $66 \%$ \\
3D-QCA MLA & $2.37 \mathrm{~mm}^{2}$ & $73 \%$ & $71 \%$ & 0.77 & $73 \%$ \\
IVUS MLA & $3.01 \mathrm{~mm}^{2}$ & $71 \%$ & $65 \%$ & 0.73 & $68 \%$ \\
\hline
\end{tabular}

FFR, fractional flow reserve, QCA, quantitative coronary angiography; MLA, minimum lumen area; IVUS, intravascular ultrasound. 


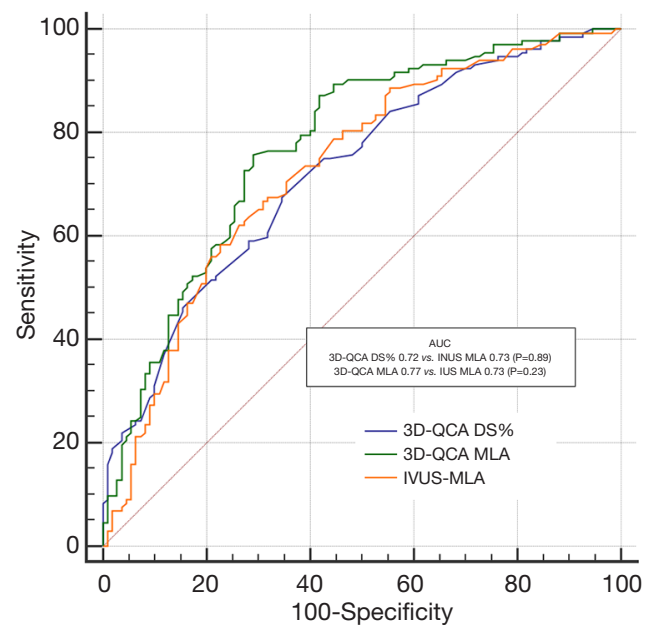

Figure 6 Comparison of ROC curves of 3D-QCA DS\%, 3D-QCA MLA and IVUS MLA for predicting FFR <0.80. QCA, quantitative coronary angiography; MLA, minimum lumen area; IVUS, intravascular ultrasound; FFR, fractional flow reserve.

\section{Limitations}

There are several limitations in our study. First, though more patients and lesions were enrolled in this study than by T. Nishi's, the present study is a retrospective, observational study with a small number of individuals. Second, patients and clinical factors were not taken into account for analysis. Third, functional ischemia is determined by not only anatomical stenosis, but also location of lesion, burden of myocardial mass, microvascular resistance, and clinical situation $(24,25)$. However, this study did not reflect the above factors. This may be why the correlation between anatomic value of 3D-QCA MLA and IVUS MLA was slightly better than that with the physiologic value of FFR.

\section{Conclusions}

3D-QCA is useful and comparable to IVUS in assessment of functionally significant coronary lesions. When IVUS or FFR are not available or are contraindicated, 3D-QCA may be a good alternative to facilitate decision making.

\section{Acknowledgments}

Funding: None.

\section{Footnote}

Reporting Checklist: The authors have completed the STARD reporting checklist. Available at http://dx.doi.org/10.21037/ cdt-20-560

Data Sharing Statement: Available at http://dx.doi. org/10.21037/cdt-20-560

Peer Review File: Available at http://dx.doi.org/10.21037/ cdt-20-560

Conflicts of Interest: All authors have completed the ICMJE uniform disclosure form (available at http://dx.doi. org/10.21037/cdt-20-560). The authors have no conflicts of interest to declare.

Ethical Statement: The authors are accountable for all aspects of the work in ensuring that questions related to the accuracy or integrity of any part of the work are appropriately investigated and resolved. The study was conducted in accordance with the Declaration of Helsinki (as was revised in 2013) and the Harmonized Tripartite Guideline for Good Clinical Practice from the International Conference on Harmonization. This study was reviewed and approved by Ajou University Hospital Institutional Review Board (AJIRB-MED-MDB-20). Because of the retrospective nature of the research, the requirement for informed consent was waived.

Open Access Statement: This is an Open Access article distributed in accordance with the Creative Commons Attribution-NonCommercial-NoDerivs 4.0 International License (CC BY-NC-ND 4.0), which permits the noncommercial replication and distribution of the article with the strict proviso that no changes or edits are made and the original work is properly cited (including links to both the formal publication through the relevant DOI and the license). See: https://creativecommons.org/licenses/by-nc-nd/4.0/.

\section{References}

1. Pijls NH, De Bruyne B, Peels K, et al. Measurement of fractional flow reserve to assess the functional severity of coronary-artery stenoses. N Engl J Med 1996;334:1703-8. 
2. Parikh RV, Liu G, Plomondon ME, et al. Utilization and Outcomes of Measuring Fractional Flow Reserve in Patients With Stable Ischemic Heart Disease. J Am Coll Cardiol 2020;75:409-19.

3. Kang SJ, Lee JY, Ahn JM, et al. Validation of intravascular ultrasound-derived parameters with fractional flow reserve for assessment of coronary stenosis severity. Circ Cardiovasc Interv 2011;4:65-71.

4. Ben-Dor I, Torguson R, Deksissa T, et al. Intravascular ultrasound lumen area parameters for assessment of physiological ischemia by fractional flow reserve in intermediate coronary artery stenosis. Cardiovasc Revasc Med 2012;13:177-82.

5. Stankovic G, Dobric M. Intravascular ultrasound and fractional flow reserve in assessment of the intermediate coronary stenosis: what you see is not what you get. J Am Coll Cardiol 2013;61:924-5.

6. Naganuma T, Latib A, Costopoulos C, et al. The role of intravascular ultrasound and quantitative angiography in the functional assessment of intermediate coronary lesions: correlation with fractional flow reserve. Cardiovasc Revasc Med 2014;15:3-7.

7. Voros S, Rinehart S, Vazquez-Figueroa JG, et al. Prospective, head-to-head comparison of quantitative coronary angiography, quantitative computed tomography angiography, and intravascular ultrasound for the prediction of hemodynamic significance in intermediate and severe lesions, using fractional flow reserve as reference standard (from the ATLANTA I and II Study). Am J Cardiol 2014;113:23-9.

8. Yong AS, Ng AC, Brieger D, et al. Three-dimensional and two-dimensional quantitative coronary angiography, and their prediction of reduced fractional flow reserve. Eur Heart J 2011;32:345-53.

9. Porto I, Dato I, Todaro D, et al. Comparison of two- and three-dimensional quantitative coronary angiography to intravascular ultrasound in the assessment of intermediate left main stenosis. Am J Cardiol 2012;109:1600-7.

10. Tu S, Barbato E, Koszegi Z, et al. Fractional flow reserve calculation from 3-dimensional quantitative coronary angiography and TIMI frame count: a fast computer model to quantify the functional significance of moderately obstructed coronary arteries. JACC Cardiovasc Interv 2014;7:768-77.

11. Nishi T, Kitahara H, Fujimoto Y, et al. Comparison of 3-dimensional and 2-dimensional quantitative coronary angiography and intravascular ultrasound for functional assessment of coronary lesions. J Cardiol 2017;69:280-6.
12. Ding D, Yang J, Westra J, et al. Accuracy of 3-dimensional and 2-dimensional quantitative coronary angiography for predicting physiological significance of coronary stenosis: a FAVOR II substudy. Cardiovasc Diagn Ther 2019;9:481-91.

13. Masdjedi K, van Zandvoort LJC, Balbi MM, et al. Validation of a three-dimensional quantitative coronary angiography-based software to calculate fractional flow reserve: the FAST study. EuroIntervention 2020;16:591-9.

14. Chung WY, Choi BJ, Lim SH, et al. Three dimensional quantitative coronary angiography can detect reliably ischemic coronary lesions based on fractional flow reserve. J Korean Med Sci 2015;30:716-24.

15. Ben-Dor I, Torguson R, Gaglia MA Jr, et al. Correlation between fractional flow reserve and intravascular ultrasound lumen area in intermediate coronary artery stenosis. EuroIntervention 2011;7:225-33.

16. Han JK, Koo BK, Park KW, et al. Optimal intravascular ultrasound criteria for defining the functional significance of intermediate coronary stenosis: an international multicenter study. Cardiology 2014;127:256-62.

17. Waksman R, Legutko J, Singh J, et al. FIRST: Fractional Flow Reserve and Intravascular Ultrasound Relationship Study. J Am Coll Cardiol 2013;61:917-23.

18. Nascimento BR, de Sousa MR, Koo BK, et al. Diagnostic accuracy of intravascular ultrasound-derived minimal lumen area compared with fractional flow reserve--metaanalysis: pooled accuracy of IVUS luminal area versus FFR. Catheter Cardiovasc Interv 2014;84:377-85.

19. Park SJ, Ahn JM, Kang SJ, et al. Intravascular ultrasoundderived minimal lumen area criteria for functionally significant left main coronary artery stenosis. JACC Cardiovasc Interv 2014;7:868-74.

20. Yang HM, Tahk SJ, Lim HS, et al. Relationship between intravascular ultrasound parameters and fractional flow reserve in intermediate coronary artery stenosis of left anterior descending artery: intravascular ultrasound volumetric analysis. Catheter Cardiovasc Interv 2014;83:386-94.

21. Koo BK, Yang HM, Doh JH, et al. Optimal intravascular ultrasound criteria and their accuracy for defining the functional significance of intermediate coronary stenoses of different locations. JACC Cardiovasc Interv 2011;4:803-11.

22. Zhang YJ, Zhu H, Shi SY, et al. Comparison between twodimensional and three-dimensional quantitative coronary angiography for the prediction of functional severity in true bifurcation lesions: Insights from the randomized 
DK-CRUSH II, III, and IV trials. Catheter Cardiovasc Interv 2016;87 Suppl 1:589-98.

23. Huang J, Emori H, Ding D, et al. Comparison of Diagnostic Performance of Intracoronary Optical Coherence Tomography-based and Angiography-based Fractional Flow Reserve for Evaluation of Coronary Stenosis. EuroIntervention 2020;16:568-76.

24. Seo KW, Lim HS, Yoon MH, et al. The impact of microvascular resistance on the discordance between

Cite this article as: Lee J, Seo KW, Yang HM, Lim HS, Choi BJ, Choi SY, Tahk SJ, Yoon MH. Comparison of three-dimensional quantitative coronary angiography and intravascular ultrasound for detecting functionally significant coronary lesions. Cardiovasc Diagn Ther 2020;10(5):12561263. doi: $10.21037 / \mathrm{cdt}-20-560$ anatomical and functional evaluations of intermediate coronary disease. EuroIntervention 2017;13:e185-92.

25. Yoon MH, Tahk SJ, Lim HS, et al. Myocardial Mass Contributes to the Discrepancy Between Anatomic Stenosis Severity Assessed by Intravascular Ultrasound and Fractional Flow Reserve in Intermediate Lesions of the Coronary Artery. Catheter Cardiovasc Interv 2018;91:182-91. 